\title{
Abstract
}

\section{Randomized double blind study on efficacy of intralesional metronidazole vs intralesional sodium stibogluconate in cutaneous leishmaniasis}

Kellapatha IP ${ }^{1}$, Wijesinghe WAMS ${ }^{2}$, Ranatunga RAWC ${ }^{1}$, Athapathu $\mathrm{W}^{3}$, Bandara MRCK ${ }^{4}$, Sumanasena $\mathrm{JAMB}^{1}$, Madarasingha $\mathrm{NP}^{1^{*}}$

${ }^{1}$ Dermatology Unit, Teaching Hospital Anuradhapura, Sri Lanka

${ }^{2} \mathrm{MOH}$ Office NPE, Anuradhapura, Sri Lanka

${ }^{3}$ Teaching Hospital Anuradhapura, Sri Lanka

${ }^{4}$ Eye Hospital Colombo

\begin{abstract}
Background

Sodium stibogluconate is considered as the gold standard treatment for cutaneous leishmaniasis. Poor response to Sodium stibogluconate has been identified in Sri Lanka highlighting the need for alternative treatments.

Methods

A Randomized, double blind clinical trial was conducted at Dermatology unit, Teaching Hospital Anuradhapura to assess the efficacy of intra-lesional metronidazole in Leishmania donovani cutaneous leishmaniasis (CL). Patients were randomly allocated to a test group which received intra-lesional Metranidazole and to the control group which received Sodium stibogluconate (SSG) weekly for 8 sessions. Patients were reviewed at intervals for 6 months to document the treatment response and for further 6 months to identify relapses. The cure rate at 12 and 24 weeks were assessed using a stratified analysis with chi- squared test.

Results

Out of 100 study participants, 53 who received SSG and 47 who received metranidazole completed the observational period. $39.6 \%$ of SSG group and $14.8 \%$ of metranidazole group had achieved complete response at 12 weeks. The complete cure rate further increased to $66.03 \%$ and $29.78 \%$ at 24 weeks respectively ( $>0.05$ ). There were two relapses at further 6 months observational period in SSG group but the relapse rate was zero for metranidazole group.

Conclusions

The standard treatment SSG shows superior efficacy to metranidazole in treating CL caused by leishmania donaovani in Sri Lanka. However Intra-lesional metranidazole showed $29.78 \%$ response rate with no relapses indicating it can be used as an alternative treatment.
\end{abstract}

Key words: Cutaneous Leishmaniasis; Sodium Stibogluconate; Metronidazole

Copyright: (C) 2015 Kellapatha IP et al. This is an open access article distributed under the Creative Commons Attribution License, which permits unrestricted use, distribution, and reproduction in any medium, provided the original work is properly cited.

\footnotetext{
*Correspondence : nayanimadara@yahoo.com
}

Cite this abstract as: Kellapatha IP, Wijesinghe WAMS, Ranatunga RAWC, Athapathu W, Bandara MRCK, Sumanasena JAMB, Madarasingha NP. Randomized double blind study on efficacy of intralesional metronidazole vs intralesional sodium stibogluconate in cutaneous leishmaniasis.Anuradhapura Medical Journal 2015;9 (2Supp):S03. DOI: http://dx.doi.org/10.4038/amj.v9i2Supp.7552 


\section{Submit your next Manuscript to} Anuradhapura Medical Journal 